\title{
Empresa pública y minería en el Sahara Occidental: Fosfatos de Bu Craa S.A., 1969-1983
}

\author{
J. M. Martínez Milán \\ Universidad de Las Palmas de G.C., Facultad de Economía, Empresa y Turismo, Departamento de Ciencias Históricas. \\ Campus de Tafira. C/ Saulo Torón 4 (Módulo E), 35017 - Las Palmas de G.C \\ jesus.martinez@ulpgc.es
}

\begin{abstract}
RESUMEN
En un contexto dominado por la rivalidad hispano marroquí en torno a la cuestión del Sahara Occidental, el estado español, a través del INI, decidió en 1969 continuar con el proyecto iniciado por Enminsa en el complejo minero de Bu-Craa, creando una nueva empresa: Fosbucraa. En este artículo pretendo analizar las dificultades financieras y técnicas por las que atravesó Fosbucraa, su estrategia comercial en el mercado mundial de fosfatos y las nefastas consecuencias que tuvo para la sociedad la precipitada salida española del Sahara. En el texto sostengo que a pesar de solventar todos sus problemas en un corto período de tiempo, la empresa vio frustrado su desarrollo por el conflicto armado entre Marruecos y el Frente Polisario, y el inicio de un período marcado por la caída de la cotización internacional del fosfato, que provocó la parálisis de la actividad minera en los años siguientes.
\end{abstract}

Palabras clave: Empresas mineras, fósforo, historia contemporánea, inversión económica, Sahara occidental.

\section{A public company and mining in the Western Sahara: Fosfatos de Bu Craa S.A., 1969-1983}

\begin{abstract}
In a context dominated by the rivalry between Spain and Morocco for the Western Sahara, the Spanish state, through the Spanish National Institute of Industry (INI), decided in 1969 to continue with the project initiated by Enminsa in the mining extraction centre of Bu-Craa by creating a new company: Fosbucraa. In this paper, I intend to analyze the financial and technical difficulties of Fosbucraa went through, its business strategy in the phosphate world market and the harmful consequences that the fast withdrawal of Spain from Sahara had on society. I argue that, despite solving all their problems in a short period of time, the development of the company was thwarted due the armed conflict between Morocco and the Polisario Front, and the beginning of a period marked by the fall in international prices of phosphate, which caused the paralysis of the mining activity in the following years.
\end{abstract}

Keywords: Mining companies, phosphorus, contemporary history, economic investment, Western Sahara.

\section{ABRIDGED ENGLISH VERSION}

\section{Introduction}

This paper studies a company called FOSBUCRAA from its birth to the end of its mining activity after being sold to the Kingdom of Morocco. This was the result of the war between the Kingdom of Morocco and the Polisario Front, and was influenced by an excess of offer and the plummeting of the world price of phosphate. In a context dominated by the rivalry between Spain and Morocco in all matters related to the Western Sahara, the Spanish Government created FOSBUCRAA to mine the Bu Craa deposit after a partnership agreement with the North American company International Mineral and Chemical Corporation (IMC) to create a society and finance the investments failed. Once the initial financial issues were overcome, which delayed the mining works by a year, the company started to operate and created a cartel with one of the main phosphate 
Martínez Milán, J. M., 2017. Empresa pública y minería en el Sahara Occidental: Fosfatos... Boletín Geológico y Minero, 128 (4): $913-929$

dealers in the world market, the Moroccan Office Chérifien des Posphates (OCP). Between them, they divided part of the European market, including Spain.

After Spain's fast withdrawal from the Western Sahara, the company entered a period of financial decline, which set its short and medium-term future: a halt in its mining activities, the impossibility to confront the financial burdens and a lack of collusion of interests between the two partners of the company: the OCP and the INI (Spanish National Institute of Industry).

\section{Results and discussion}

Morocco subordinated economic interests (fishing and phosphates) to its territorial interests, not only to achieve the annexation of the Western Sahara, but as a means of pressurizing the first democratic governments in Spain after the death of Franco, in order to break the neutrality with regard to the former Spanish colony. The company's lack of a financial policy from the Spanish Government delayed the beginning of FOSBUCRAA's mining activity until 1973. However, in the previous years (1971 and 1972) the State acted appropriately to solve this situation (Fig. 1). On the one hand, it approved the company's investment, financing and profitability programme (Table 1) allowing the society to resort to the capital market to gain foreign resources and use the INI as the guarantor of credits granted by suppliers. On the other hand, it settled the commercial policy to be followed regarding the two main natural phosphate producing and export companies worldwide: the OCP and the IMC.

A year after it started trading (1974), the production of phosphate (Table 2) had not reached the figures set out in that year's forecast. However, the perspectives of achieving the production objectives in the following years were quite favourable despite the problems encountered: an excess of chlorine in the phosphate rock mineral, technical downtimes in the extraction of the mineral and at the treatment plant, and the attack carried out by the Polisario Front, which affected various parts of the conveyor belt.

In an oligopolistic market, where the IMC and the OCP were the two main players, the INI decided that FOSBUCRAA should enter a partnership with the latter, due to its European presence, and reach temporary agreements with the IMC in South American and Asian markets, where the Moroccan company had no market. The Spanish-Moroccan cartel began its activity in 1972. A year later, the rocketing of the worldwide price of phosphate (Table 3) led Spanish fertiliser companies to threaten the Government with transferring those price increases to the final price of phosphate fertilisers. Despite FOSBUCRAA's administration counsel's denial, the Spanish government made the company sell most of its production to the Spanish market (Table 4) at a price below the market price (Table 5). This was harmful to the Spanish company as it decreased its availability for foreign markets and endangered the agreement entered with the OCP regarding the domestic market supply, as well as being economically damaging for the company. However, in a context marked by high phosphate prices worldwide, the company was able to achieve its goals and profits in its income statement (Table 6).

Spain's fast withdrawal from the Sahara and the beginning of a period influenced by the fall in price of phosphate (Fig. 2) slowed down the company's economic activity in 1975, as well as the INI's decision to leave the society. However, Morocco made the Institute remain as a shareholder, as they did not want to buy all the participations during the negotiations, which ended with a purchase agreement of $65 \%$ of the shares by the OCP, while the Institute kept 35\%, impeding any kind of capital increase by the main partner. The headquarters were then moved to Rabat and the OCP took over the company.

The war between the Polisario Front and the Kingdom of Morocco in 1976 seriously damaged the mineral extraction centre to the extent that it halted its economic activity in April of the same year. The damages occasioned by the war and the increase in the maintenance and labour costs, together with the company's fragile financial situation, worsened throughout the years without restarting its business activity. Between 1976 and 1981, the total loss reached 27,000 million pesetas (Fig. 3). The situation was alarming for the OCP, which was covering the yearly losses with treasury credits, and the INI, which was absorbing private companies facing bankruptcy, under governmental orders, in order to slow down the unemployment rate during the Spanish transition to democracy. A new agreement between the two partners was entered in 1980 in order to try to solve these problems, by which the company would delay, for three years, the repayment of capital plus interests from loans taken by the company from banks, the Institute and the OCP.

However, the situation only worsened during those three years. Although the company had opened a new mining site during the summer of 1982, the company's financial situation created irreconcilable differences between the partners on solving the debt issues. The OCP rejected paying for FOSBUCRAA's annual losses by itself, asking for an increase of the partnership capital to put its accounts in order. The INI, however, wanted the OCP to pay its share of the debt, in dollars and pesetas, which FOSBUCRAA had contracted since 1974 with a group of international banks (co-signed by the INI) and the Institute itself, which in 1983 made up a total of 23,601 million pesetas. In addition, the Institute claimed for Spanish governmental help so that, in the 
adverse event, it would grant a State-to-State loan in order that the Kingdom of Morocco could pay the amount, as the INI was having difficulties at the time. In the event that the OCP continued with its refusal and the Institute's administration body requested the Spanish government to authorise the guarantee of such loans.

The two governments kept their stances and, in the end, the weight of Spanish political and economic interests in the Kingdom of Morocco resulted in determining the decision taken by the government in Madrid. In 1996, and in line with the stance defended by the OCP, a capital increase was approved. The Institute did not participate and its shares were lowered to 12 percent. Six years later (2002), the Spanish Society of Industrial Participations (SEPI), which succeeded the INI, sold the remaining shares and abandoned being a FOSBUCRAA shareholder.

\section{Introducción}

Tras el descubrimiento de la existencia de fosfatos en el Sahara Occidental, realizado por el geólogo Manuel Alía Medina entre los años 1945 y 1947, el gobierno español encomendó a la Empresa Nacional "ADARO" de Investigaciones Mineras S.A. (ENADIMSA) la investigación de la zona fosfatada con el fin de evaluar sus reservas y su cubicación. Dada las escasas inversiones y la zona elegida (río Abadila y meseta de Izic, próximos a la costa del Aaiún), el fosfato encontrado era de una calidad muy baja (inferior al $45 \%$ BPL), lo que hizo inviable su explotación y comercialización. Después de un breve período de tiempo en el que el proceso se estancó, el gobierno volvió a reactivar su interés por los fosfatos del Sahara con la constitución, a mediados de 1962, de la Empresa Nacional Minera del Sahara S.A. (ENMINSA), la cual emprendió las investigaciones que culminaron un año más tarde con la localización y cubicación del yacimiento de Bu Craa. A mediados de los años sesenta, el estado franquista intentó alcanzar un acuerdo con compañías estadounidenses para compartir el excesivo coste financiero que suponían las fuertes inversiones a realizar en el citado yacimiento. Ante el fracaso, el gobierno decidió dar un paso adelante y finalizar, motu proprio, una obra que había puesto en marcha años antes. Con ese fin creó Fosfatos de Bu-Craa S.A. (FOSBUCRAA), nombre que adoptó ahora la empresa Enminsa, cambió el consejo de administración y amplió el capital social escriturado.

Los hechos aquí narrados se basan en la documentación de la propia empresa, depositada en el Centro de Documentación y Archivo Histórico de la Sociedad Estatal de Participaciones Industriales en Madrid (ASEPI), que recoge los fondos del desaparecido INI; y en la documentación de la embajada francesa en Rabat, que se conserva en el Centre des Archives Diplomatiques de Nantes (CADN).

El trabajo está estructurado en cuatro apartados. En el primero se aborda la evolución de los intereses hispano-marroquíes en el Sahara Occidental. En el segundo se analizan las dificultades financieras de la empresa en los primeros años de existencia y su entrada en explotación, marcada por el final de la presencia española en la colonia. En el tercero se estudia la creación de un cártel hispano-marroquí en un mercado mundial claramente oligopolístico. En el cuarto y último se examinan las consecuencias de la precipitada salida de España del territorio, con la venta de la mayoría de las acciones a Marruecos y los efectos de la parálisis de la actividad económica de la empresa a raíz de la guerra entre el Frente para la Liberación de Saguia al-Hamra y Río de Oro (FRENTE POLISARIO) y las Fuerzas Armadas Marroquíes (FAR), y del comienzo de una coyuntura internacional marcada por la baja cotización del fosfato.

\section{El Sahara Occidental y la evolución de los intereses hispano-marroquíes, 1969-1983}

Tras la retrocesión de Sidi Ifni al reino alauita en 1969, Marruecos flexibilizó sus posiciones territoriales con respecto al Sahara Occidental, aceptando un referéndum de autodeterminación. La evolución de la posición marroquí no suponía la renuncia a su reivindicación sobre la colonia. Todo lo contrario, se trataba de una táctica dilatoria con el único fin de romper el aislamiento regional en el que el círculo vicioso de sus reivindicaciones territoriales lo había metido.

El clima de distensión regional con Argelia y Mauritania fue impulsado por el inmovilismo del gobierno español. A lo largo de los años sesenta existían dos visiones contrapuestas dentro del ejecutivo franquista a la hora de abordar que había que hacer con el territorio sahariano. Una estaba representada por el Ministerio de Asuntos Exteriores (MAE), con el ministro Castiella al frente. En su opinión había que proceder a la descolonización del territorio siguiendo los dictámenes de la ONU con objeto de salvaguardar los intereses españoles. Se seguía así el modelo francés en Mauritania. Para ello era necesario reformar 
previamente la administración del Sahara y realizar inversiones económicas que impulsaran el desarrollo del territorio. Frente a esta posición se situaban los principales organismos ministeriales, nucleados en torno a Presidencia del Gobierno. Para ellos, el Sahara no era una moneda de cambio y no se podía ir hacia una descolonización rápida ya que consideraban que la población no estaba preparada (Pardo Sanz, 2007). Con la sustitución de Fernando María Castiella por López Bravo al frente del MAE en 1969, un hombre más proclive a Carrero Blanco, las diferencias desaparecieron momentáneamente dentro del ejecutivo franquista, aunque no todos los diplomáticos opinaban lo mismo que su nuevo jefe (Piniés, 1990).

Entre 1970 y 1972, la cuestión del Sahara Occidental pasó a un segundo plano en las preocupaciones de Rabat, dada la crisis interna que atravesaba el país y el intento de magnicidio de Hassan II. Aprovechando esta coyuntura, el gobierno español intentó un acercamiento con los países de la zona, firmando acuerdos de pesca y aéreos con Marruecos e invirtiendo en la constitución de una sociedad de transformación del pescado en Mauritania (Martínez Milán, 2014). Fue en este contexto de distensión de las relaciones hispano-marroquíes, cuando el reino alauita planteó a España, a través de la Office Chérifien de Phosphates (OCP), la posibilidad de participar en el accionariado de Fosbucraa. La OCP se creó por dahir (decreto) de 7 de agosto de 1920. A esta empresa se le dotó de personalidad jurídica y autonomía financiera propia, y se le concedió el monopolio de los fosfatos en la zona del protectorado francés en Marruecos. Tras la independencia del país cherifiano en 1956, la empresa fue nacionalizada (Hatton, 2009). Hassan II utilizó a la OCP como instrumento para conseguir sus objetivos territoriales, poniendo al frente de la misma a un hombre dotado de un extraordinario olfato para los negocios y fiel a su persona: Mohamed Karim Lamrani (Dalle, 2004).

En los primeros contactos entre responsables de OCP y del INI celebrados en 1971, los representantes marroquíes exigieron al gobierno español no sólo una participación accionarial en Fosbucraa, sino en todas las riquezas mineras del Sahara; al tiempo que abogaban por la creación de una sociedad mixta para la fabricación de ácido fosfórico. Todo ello a cambio de abandonar "la postura reivindicatoria de Marruecos respecto al Sahara" (ASEPI, FOSBUCRAA, caja 44, doc. 4. MEMORANDUM. Rabat, 8 juillet 1971). $\mathrm{Ni}$ Marruecos iba a abandonar sus reivindicaciones, ni España iba ceder soberanía en el Sahara. Al igual que había ocurrido con Melilla en los años sesenta (Díaz Morlán, 2015), el gobierno español se negó a cualquier intento de marroquinizar la política del territorio y, por tanto, a cualquier posible participación de OCP en el accionariado de Fosbucraa.

Tras el asesinato de Carrero Blanco en diciembre de 1973, su sustituto, Carlos Arias Navarro, puso en marcha a principios de 1974 la elaboración de un estatuto de autonomía para el Sahara con el fin de prolongar la presencia española en el territorio todo el tiempo que fuera posible (Diego Aguirre, 1988; Rodríguez Jiménez, 2015). Ante esta noticia, Hassan II desplegó una campaña diplomática en los organismos internacionales en pro de los intereses de su país y de la anexión del territorio que dio sus frutos. La presión marroquí, la enfermedad del dictador y las contradicciones internas del régimen franquista que se debatía entre permanecer a toda costa en el Sahara y descolonizar de acuerdo con la ONU (el ministro Cortina y algunos diplomáticos en Naciones Unidas), o retirarse y ceder el territorio al reino alauita, precipitaron la salida de España y el traspaso de la administración colonial a Marruecos y la República Islámica de Mauritania, con el beneplácito de Estados Unidos y Francia (Cordero and Lemus, 2015).

En el inicio de la transición democrática, los gobiernos de Unión de Centro Democrático (UCD) optaron por una política de estricta neutralidad en todo lo atinente al Sahara Occidental, con el fin de satisfacer las expectativas de Marruecos y Argelia. Lejos de lograrlo, la situación se tornó en una presión constante por parte de ambos países para atraer a España hacia sus posiciones. El reino alauita utilizó con España la política del "palo y la zanahoria". Por un lado explotó la vulnerabilidad española amenazando con las reivindicaciones territoriales sobre Ceuta y Melilla. Por el otro, apoyó la españolidad de las islas Canarias en el debate sobre la africanidad del archipiélago en el seno de la Organización de la Unidad Africana (OUA).

Al igual que hizo en 1971, el reino alauita no tuvo ningún problema en subordinar sus intereses económicos al servicio de sus intereses territoriales. En las contrapartidas secretas otorgadas por Marruecos a España en materia pesquera en los acuerdos tripartitos de Madrid de 1975, figuraba la concesión de derechos de pesca durante un período de 15 a 20 años para unos 800 barcos españoles en aguas del mediterráneo, del océano atlántico y del caladero sahariano. Rabat entendía que los beneficios económicos que esos derechos reportarían a España tenían que ser compensados con el reconocimiento de la soberanía marroquí sobre la excolonia española. Sin embargo, el gobierno de UCD se negó al no aceptar la distinción que se hacía entre aguas de soberanía marroquí al norte de cabo Nun y de administración 
marroquí al sur del mismo, generando una situación conflictiva para los pesqueros españoles hasta que se firmó un acuerdo definitivo en 1983. Otro tanto de lo mismo ocurrió con los fosfatos. En los acuerdos de Madrid, el gobierno marroquí se opuso a adquirir la totalidad de las acciones de Fosbucraa. Con ello obligó al INI a permanecer en el accionariado de la empresa, mostrándolo como un ejemplo de la colaboración hispano-marroquí en el Sahara. En ese intento de buscar el acercamiento español a sus posiciones, Rabat no tuvo reparos en adjudicar a Enadimsa, en 1976, la investigación y cubicación del nuevo yacimiento de fosfato de Meskala, próximo a Mogador.

Con la llegada del Partido Socialista Obrero Español (PSOE) al gobierno a finales de 1982, el ejecutivo socialista, a pesar de mantener la neutralidad en la cuestión del Sahara, puso en marcha una política de colaboración con el Magreb, al considerar esta región como una zona de desequilibrios a la que había que ayudar política y económicamente. Los resultados de esta política no empezaron a vislumbrarse hasta finales de la década (Larramendi, 1997).

\section{El impulso definitivo, 1969-1975}

Fosfatos de Bu-Craa S.A. (FOSBUCRAA) nació formalmente el 22 de mayo de 1969, fecha en la cual quedó registrada la escritura de ampliación de capital y modificación otorgada por la Empresa Nacional Minera del Sahara, S.A. (ENMINSA), ante el notario de Madrid, Antonio Yáñez Cancio. Además del cambio de denominación, del aumento del capital social a 5.000 millones de pesetas, del traslado del domicilio social a Las Palmas de Gran Canaria y de la posibilidad de ampliar el número de consejeros, incluyendo a ciudadanos saharauis; el objeto de la empresa se fijó en la explotación, concentración y comercialización de los minerales existentes en yacimientos de fosfatos y en todas aquellas actividades relacionadas directa o indirectamente con estos (ASEPI, FOSBUCRAA, caja 4515, doc. 28. Escritura de ampliación de capital y modificación de Sociedad, otorgada por Enminsa. Madrid, 22 de mayo de 1969).

Fosbucraa heredó de Enminsa las dificultades financieras nacidas tras el fracaso de las negociaciones entabladas en 1966 y 1967 entre el Estado español y firmas norteamericanas (Ríos, 1989; Martínez Milán, 2017; Camprubí, 2015). En aquellos años se consideró que la aportación de medios económicos externos al INI, mediante participación en el capital social, haría innecesario cualquier otra previsión de recursos financieros. En consecuencia, la financiación de Enminsa y posteriormente de Fosbucraa no se incluyó en las previsiones del II Plan de Desarrollo (1969-1972), ni en el plan cuatrienal del INI. Ante estas circunstancias, la empresa remitió al INI, en el verano de 1969, un "Programa de Inversiones, Financiamiento y Rentabilidad de la empresa Fosbucraa S.A.," que el Instituto elevó al Gobierno para su estudio y aprobación. La empresa sugería que para resolver de modo satisfactorio el plan de inversiones se optara por dos vías:

a) Recursos propios más préstamos obtenidos por el INI en el mercado de capitales nacional e internacional.

b) Autorizar al Instituto a firmar avales correspondientes a los créditos concedidos por los proveedores hasta un máximo anual de 3.400 millones de pts. (ASEPI, FOSBUCRAA, caja 4.515, doc. 21. Programa de Inversiones, Financiamiento y Rentabilidad de Fosbucraa. Madrid, 31 de julio de 1969).

No se pedía nada nuevo, sólo adaptar la financiación de la empresa a la manera en la que se financiaban las empresas del INI entre mediados de los años sesenta y setenta. Esto es: ampliaciones de capital social, créditos y préstamos a medios y largo plazo, y avales para la obtención de préstamos en el mercado nacional e internacional (Martín Aceña and Comín, 1991).

El gobierno, empero, no adoptó resolución alguna sobre la propuesta y el INI fue resolviendo de "modo perentorio" los problemas financieros de Fosbucraa con medidas sustitutivas que se mostraron insuficientes. Esto provocó que la entrada en explotación de la empresa prevista para enero de 1972 se retrasara un año. Las causas estaban estrechamente relacionadas con el retraso en la obtención de avales por parte del Instituto, que había obligado a paralizar obras comprometidas en los depósitos reguladores y en las plantas de tratamiento, y con la paralización desde mediados de 1969 de la financiación de bienes de equipo de fabricación española a través del Instituto de Crédito a Medio y Largo Plazo (ICMLP). En un intento de tranquilizar al consejo de administración de Fosbucraa y al propio INI, el 17 de noviembre de 1970 se celebró una reunión en Presidencia del Gobierno, en la que se intentó tranquilizar a los representantes del Instituto, argumentando que los problemas financieros de la sociedad formaban parte de los del propio INI. "Una vez resuelta la financiación de éste, se resuelve la de Fosbucraa". El encuentro acabó con un acuerdo para activar al máximo las inversiones de la empresa, dotándola de los medios financieros para llevarlo a cabo, al tiempo que se fijaron las directrices de la política comercial. (ASEPI, FOSBUCRAA, caja 45, doc. 9. Nota informativa INI 50/70. Madrid, 19 de noviembre de 1970). 
El 28 de enero de 1971, el Instituto envió al Gobierno un nuevo "Programa de Inversiones, Financiamiento y Rentabilidad de la empresa Fosbucraa S.A." El objetivo no era otro que alcanzar los objetivos de producción y reducir gastos en el menor tiempo posible. En el nuevo programa (Tabla 1), los niveles de producción habían variado con respecto al programa de inversiones de 1969. Hasta ese momento se había considerado que el nivel de producción a alcanzar en 1973 era de 3 millones de toneladas. Ahora se aumentaba a 4 millones en ese año y en el siguiente, y a 6 y 10 millones respectivamente en 1975 y 1979. Con el fin de cumplir con el programa, el INI solicitó la autorización del Gobierno para aumentar el capital social de 5.000 millones a 6.500 millones de pesetas, que estarían desembolsados en 1974, así como para incrementar las inversiones en un $11 \%$ cada uno de los tres años con respecto a las previsiones de 1969. El aumento en ambas partidas venía justificado por los retrasos que se habían producido desde un principio, lo que implicaría unas necesidades de tesorería más elevadas de las previstas para los años 1971, 1972 y 1973, además de la imposibilidad de financiar a largo plazo los suministros españoles que estaban por contratar. Con ello pensaban que la proporción entre los fondos propios y los recursos ajenos se situaría en unos límites prudentemente razonables.

Asimismo, el Instituto demandaba que se le facultara para arbitrar las medidas oportunas con objeto de que Fosbucraa obtuviera los créditos necesarios en el mercado de capitales hasta alcanzar un saldo neto máximo de 6.791 millones de pesetas en 1974, al tiempo que concertar créditos con los proveedores por un saldo de hasta 2.616 millones de pesetas en 1972, a lo que habría que sumar otros cuatro mil millones más entre 1973 y 1974. Cuanto más se tardara en solucionar el tema de la financiación, más

\begin{tabular}{|c|c|c|c|c|}
\hline Año & Producción & $\begin{array}{c}\text { Inversiones } \\
\mathbf{1 9 7 0}\end{array}$ & $\begin{array}{c}\text { Inversiones } \\
\mathbf{1 9 6 9}\end{array}$ & Diferencia \\
\hline 1973 & 4 & 15.173 & 13.580 & 1.593 \\
\hline 1975 & 6 & 16.985 & 15.335 & 1.650 \\
\hline 1979 & 10 & 20.840 & 19.004 & 1.836 \\
\hline
\end{tabular}

Fuente: ASEPI, FOSBUCRAA, caja 4902, doc. 46. Programa de Inversiones, Financiamiento y Rentabilidad de la Empresa Fosfatos de Bu-Craa S.A. Madrid, 28 de enero de 1971

Tabla 1. Inversiones previstas en relación con la capacidad de producción anual, 1973-1979 (millones de pts y millones de toneladas). Table 1. Investments planned in connection with the annual production capacity, 1973-1979 (millions of pesetas and millions of tons). habría que subir el techo mínimo de las inversiones para no deteriorar la situación financiera de la empresa y su posible rentabilidad. A las medidas financieras habría que añadir el deseo del consejo de administración de regularizar cuanto antes la situación administrativa de la concesión minera. El consejo de ministros celebrado el 9 de julio de 1971 aprobó, por fin, el programa de inversiones y financiación presentado por Fosbucraa.

La actividad minera arrancó en 1969 con la formalización y adjudicación de los contratos de las instalaciones que estaban pendientes cuando Fosbucraa tomó el relevo de Enminsa: dragalina, planta de tratamiento, sistema de transporte, conjunto de calderas, turbina y planta potabilizadora. De igual modo se procedió a definir el método de enriquecimiento del mineral. El mineral extraído de la mina se llevaría a una planta de tratamiento primaria, ubicada en las proximidades de $\mathrm{Bu} \mathrm{Craa}$, donde se procedería a la trituración y cribado a $10 \mathrm{~mm}$ con el fin de eliminar los elementos estériles gruesos y facilitar el transporte a través de la cinta hasta la planta de tratamiento principal situada en la playa del Aaiún. Allí se realizaría una clasificación granulométrica por vía húmeda, disgregación y limpieza del mineral para eliminar impurezas (cloruro) y el agua empleada en el proceso.

En cuanto al transporte del mineral, el consejo de administración de Fosbucraa aprobó algunas modificaciones relacionadas con la longitud y trazado de la cinta transportadora que ya estaban previstas en el contrato firmado en enero. En primer lugar, modificaron los giros en grados en algunos tramos. En segundo lugar, alargaron la cinta en 3.1 kilómetros por el extremo de la mina y lo acortaron en 1 kilómetro por el extremo de la costa. De esta manera la cinta de transporte tendría una longitud de 98.65 kilómetros, dividido en tres alineaciones rectas. La primera, que iría desde la mina hasta Uad el Jat (río el Jat) (33.514 metros), tendría cuatro tramos. La segunda, que cruzaría el citado río, estaría compuesta por dos tramos (17.706 metros) y la última, que completaría el trayecto desde el Uad el Jat hasta la costa (47.432 metros), estaría formado por cinco tramos (Figura 1). En total, la cinta tendría once tramos. En lo atinente a las obras auxiliares, a finales de 1969 quedó finalizada la obra civil marítima del cargadero de mineral, iniciándose las obras del edificio principal de la central eléctrica y una subestación (ASEPI, FOSBUCRAA, caja 4728. Resumen anual de trabajos. Madrid, 1969).

A lo largo de 1970, y como consecuencia de las dificultades financieras descritas más arriba, no se realizó ningún montaje en las instalaciones. La única obra a reseñar ese año fue la estabilización de la 


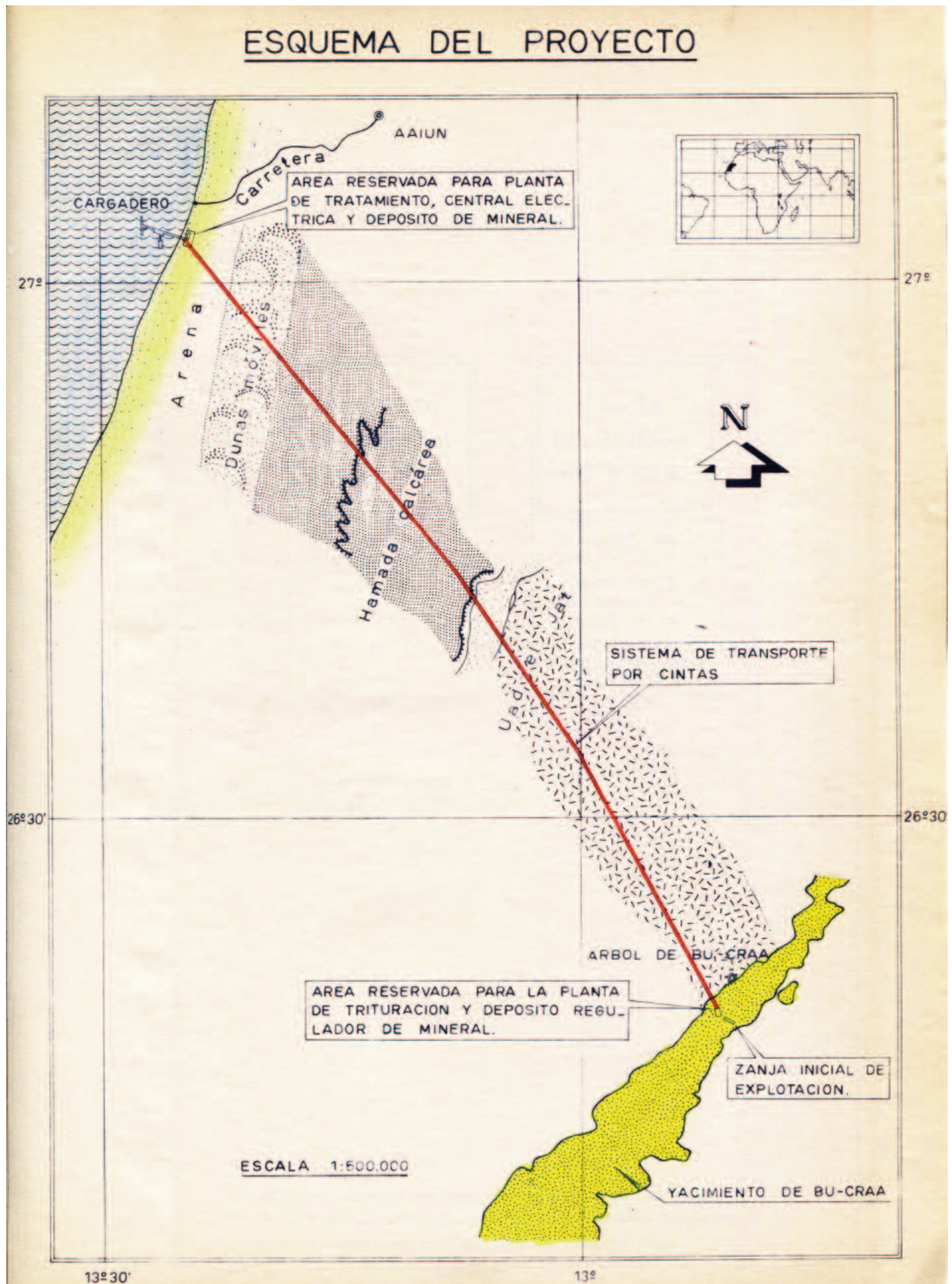

Figura 1. Mapa litológico para la geotecnia de la cinta transportadora. Reproducido con permiso de la SEPI.

Figure 1. Lithological map for the geotechnics of the conveyor belt. Reproduced with authorization from SEPI. 
cadena de dunas cercana a la costa, llevada a cabo por Esso Standar Española, en la parte en que era atravesada por el sistema de cintas. Mediante el riego a presión de productos petrolíferos se estabilizó un frente de 9 kilómetros de largo y 250 metros de ancho (ASEPI, FOSBUCRAA, caja 4904. Resumen anual de trabajos. Madrid, 1970).

En 1971, y gracias a la aprobación del programa de inversiones y financiamiento por parte del Gobierno, las obras volvieron a recobrar el impulso, poniéndose en marcha todos los proyectos que estaban paralizados. Un año después (1972) se terminaron de montar la mayor parte de las instalaciones productivas que formaban el complejo minero. El único retraso seguía estando en las plantas de tratamiento mineral. El resultado, ya conocido, fue la demora en un año de la puesta en marcha del complejo, que se tradujo en una falta de liquidez muy por encima de lo esperado, teniendo que cubrirse con un crédito a corto plazo solicitado a la banca nacional (ASEPI, FOSBUCRAA, Memoria y Balance del ejercicio 1972. Madrid).

En 1973 se produjo por fin la entrada en explotación de Fosbucraa. Durante ese año, la producción de mineral pretratado y apilado en Bu Craa superó el millón de toneladas, alcanzándose una producción de fosfato concentrado de 70.2248 toneladas de calidades $75 \%$ y $80 \%$ BPL (Tabla 2 ).

La capacidad de producción de mineral preconcentrado en 1974 creció un $247 \%$ con respecto a 1973 , mientras que la producción de mineral concentrado lo hizo un $329 \%$, alcanzando los tres millones de toneladas, un millón menos de lo previsto en el programa de inversiones y financiamiento de 1971. Uno de los problemas que más acuciaba a los técnicos de la empresa era el alto contenido en cloro del fosfato concentrado. Cuando se montaron las instalaciones, el cloro no estaba penalizado en los mercados internacionales. La introducción de nuevos procesos de fabricación en la industria de fertilizantes, especialmente en la fabricación de fertilizantes no agrícolas

\begin{tabular}{|c|c|c|c|}
\hline & $\mathbf{1 9 7 3}$ & $\mathbf{1 9 7 4}$ & $\mathbf{1 9 7 5}$ \\
\hline Arranque estéril & 4.200 .000 & 9.271 .405 & 15.934 .553 \\
\hline Apilado Bu-Craa & 1.060 .000 & 3.680 .013 & 5.627 .905 \\
\hline Apilado Puerto & 1.050 .000 & 3.467 .751 & 4.941 .366 \\
\hline Producción Planta & 702.248 & 3.012 .172 & 2.801 .398 \\
\hline
\end{tabular}

Tabla 2. Actividad productiva de Fosbucraa, 1973-1975 ( $\mathrm{m}^{3}$ arranque y toneladas resto) (elaboración propia a partir de Memorias y Balances de Fosbucraa).

Table 2. Fosbucraa productive activity, 1973-1975 ( $\mathrm{m}^{3}$ start and tone rest) (Own elaboration from annual reports and balance sheets of Fosbucraa). (detergentes), exigió la reducción de cloro a niveles muy bajos en el mineral concentrado. Este problema no se resolvió del todo hasta 1975, gracias a la instalación de ocho filtros planos y treinta dos centrífugas en la planta de producción (ASEPI, FOSBUCRAA, caja 5.557, doc. 14. Consejo de Administración. Acta $\mathrm{n}^{\circ}$ 173. Madrid, 29 de abril de 1975. Y, Memoria y Balance de Fosbucraa, 1975).

Además de esta circunstancia, la empresa tenía otras dos cuestiones a las que hacer frente: la falta de personal cualificado y la carencia de un sistema ágil de gestión de los stocks. Entre 1969 y 1972, último año en el que la empresa nos ofrece datos de los empleados en función de su cualificación, el número de ingenieros se mantuvo estancado en torno al $17 \%$ de media en ese período. La lejanía de la península y la falta de incentivos para trasladar a las familias a un entorno desértico encarecían el coste de la mano de obra más cualificada. En las conclusiones de un estudio elaborado en mayo de 1974 por el ingeniero de minas de la división de producción, Antonio García Moreno, se advertía de que en tanto no se resolvieran estas dos cuestiones, la producción de la planta seguiría oscilando entre las 150.000 y las 200.000 toneladas mensuales, con tendencia a disminuir. Por el contrario, con unas condiciones de servicio correctas, en la planta de tratamiento se podría alcanzar una producción mensual entre las 200.000 y las 250.000 toneladas (ASEPI, FOSBUCRAA, caja 2383. doc. 23. Consejo de Administración. Acta $n^{\circ} 161$. Madrid, 28 de mayo de 1974. Y, doc. 42. Junta General Ordinaria de Accionistas. Acta $\mathrm{n}^{\circ}$ 19. Las Palmas de G.C., 14 de junio de 1974).

Aunque la actividad productiva de la empresa fue importante en 1974, la producción de fosfato concentrado pudo haber sido mayor de la que realmente fue, si no hubieran coincidido una serie de circunstancias en el último cuatrimestre del año que provocaron una reducción de la producción anual estimada en 200.000 toneladas. En primer lugar, hay que destacar la parada de la planta de tratamiento durante tres semanas en el mes de julio, como consecuencia de una reparación general. En segundo lugar, la extracción de estéril estuvo paralizada durante mes y medio por rotura de la dragalina, dando lugar a una disminución tanto de la cantidad arrancada como de la producción de mineral. A pesar de todo, fue el atentado del 20 de octubre el que realmente mermó el nivel de producción de fosfato. En la noche del 19 al 20 de octubre, un comando de Frente Popular para la Liberación de la Sagia al-Hamra y Río de Oro (POLISARIO), del que formaban parte algunos saharauis empleados de la empresa, cometió un atentado que afectó a las estaciones de trasbordo de la cinta núme- 
ros VII y VIII, destruyendo doscientos metros de tramo de cinta de la estación VII y buena parte de sus instalaciones eléctricas, así como otros 100 metros de cinta entre las estaciones VIII y IX con todas las instalaciones eléctricas de la primera. La interrupción del transporte redujo tanto la cantidad extraída de estéril como la producción de mineral concentrado. Los directores e ingenieros de Fosbucraa buscaron una solución alternativa mientras se arreglaba el tramo de cinta dañado, cuyos daños estimaron en unos 70 millones de pesetas. La solución que encontraron fue la de poner en marcha nuevamente la cinta en los tramos no averiados y establecer un transporte discontinuo (camiones) entre las estaciones dañadas (ASEPI, FOSBUCRAA, caja 5.383, doc. 39. Consejo de Administración. Acta $\mathrm{n}^{\circ}$ 167. Madrid, 31 de octubre de 1974. Y, Bárbulo, 2002).

En 1975, la actividad económica de la empresa estuvo marcada por la tensa situación que se vivió en el Sahara Occidental. Este hecho unido al inicio de la recesión mundial en la demanda de fosfato fue el causante de la caída de la producción de mineral concentrado en un $7 \%$ con respecto a 1974 . A partir de ese momento, el consejo de administración de la empresa gestionó ante el INI la posibilidad de integrar en empresas del holding a aquellos trabajadores del centro minero del Sahara que en función de su permanencia en el territorio así lo solicitaran. El 13 de diciembre la actividad minera quedó suspendida, considerando al personal afectado "en situación equivalente a la de permiso restringido" hasta el 11 de enero, si bien el plazo fue prorrogado quince días más (ASEPI, FOSBUCRAA, caja 5557, doc. 22. Consejo de Administración. Acta $\mathrm{n}^{\circ}$ 175. Madrid, 6 de junio de 1975).

\section{El mercado mundial de fosfatos y la formación de un cártel hispano-marroquí, 1972-1975}

Entre 1950 y principios de los años sesenta el mercado mundial de fosfatos estuvo dominado por Estados Unidos y Francia. Esta última en razón de la posesión de importantes minas de roca fosfática en el Magreb y en el África subsahariana (Senegal y Togo). Durante ese período, ambos productores mantuvieron una estrategia de acuerdos comerciales que les aseguró la casi totalidad de las exportaciones mundiales con unos precios estables que rondaban de media los $6 \mathrm{u}$ 8 dólares la tonelada FOB (Anani Mensah, 2003).

A partir de 1964 y hasta 1972 la coyuntura cambió. El estancamiento de la demanda mundial y el exceso de oferta provocaron una guerra de precios entre OCP y los productores norteamericanos de Florida que permitió a estos últimos ganar terreno en el mercado mundial, duplicando sus exportaciones en el quinquenio 1964-1968 (Oualalou, 1975; Zenaidi Karray, 1987; Anani Mensah, 2003).

La situación del mercado internacional del fosfato cambió de nuevo a finales de 1972. Una serie de malas cosechas en Estados Unidos, acompañado de sequías en China y la URSS, provocaron un incremento en la demanda de cereales que hizo crecer la demanda de fertilizantes y la de mineral de roca fosfática, dando lugar a un cambio en la tendencia de los precios y al inicio del boom fosfatero que duró desde 1973 hasta finales de 1975 . Si bien la subida del petróleo y la creciente demanda de abonos fosfatados, con la entrada de nuevos consumidores (Brasil y China), contribuyó al alza del precio del fosfato, fue la subida impulsada por Marruecos en el otoño de 1973 lo que produjo un fuerte incremento de la cotización mundial del mineral de roca fosfática que, en términos nominales, se cuadruplicó en los dos años siguientes (Tabla 3). Los demás países, incluida España, siguieron la senda alcista marcada por el reino cherifiano.

EI INI deseaba que Fosbucraa entrara en el mercado asociándose con los principales productores: International Mineral and Chemical Corporation (IMC) de Estados Unidos y OCP, con el fin de minimizar riesgos en un mercado oligopolístico. IMC volvió a ponerse en contacto con el Instituto en 1971 (Camprubi, 2015). Su intención era constituir una sociedad para la venta y distribución del fosfato con sede en Paris y con un capital social de un millón de dólares, en la que Fosbucraa tendría el $51 \%$ de las acciones e IMC el $49 \%$ restante. El objeto de la sociedad era repartirse el mercado mundial en función de sus zonas naturales de comercialización (Europa y América del Sur para las exportaciones procedentes del Sahara; y Japón, Extremo Oriente y América del Norte para las de Florida), si bien obligaba a la sociedad española a pagar una comisión de un dólar por tonelada vendida, teniendo que abonar además los gastos correspondientes en caso de utilizar los servicios de distribución y comercialización de IMC. La empresa estadounidense no descartaba entrar en el accionariado de Fosbucraa, siempre que se tomaran las previsiones adecuadas para que en el caso de un eventual cambio de soberanía del territorio los intereses de la nueva sociedad y de sus accionistas quedaran debidamente protegidos. Con el fin de lograrlo, IMC propuso al INI alcanzar un acuerdo con OCP para adquirir el $50 \%$ de las acciones de Fosbucraa, idea que fue rechazada por el Instituto dada la posición del gobierno español a este respecto (ASEPI, FOSBUCRAA, caja 44, doc. 3. Presidente del INI a Ministro de Industria. Madrid, 29 de enero de 1971). 
Martínez Milán, J. M., 2017. Empresa pública y minería en el Sahara Occidental: Fosfatos... Boletín Geológico y Minero, 128 (4): $913-929$

\begin{tabular}{|c|c|c|c|c|c|c|}
\hline Países & $\mathbf{1 9 7 0}$ & $\mathbf{1 9 7 3}$ & $\mathbf{1 9 7 4}^{\mathbf{1}}$ & $\mathbf{1 9 7 4}^{\mathbf{2}}$ & $\mathbf{1 9 7 5}^{\mathbf{1}}$ & $\mathbf{1 9 7 5} \mathbf{2}^{\mathbf{2}}$ \\
\hline Marruecos (75\%) & 11,75 & 14,25 & 42,00 & 63,00 & $\mathbf{6 8 , 0 0}$ & $\mathbf{6 8 , 0 0}$ \\
\hline Marruecos (80\%) & nd & nd & 50,00 & 75,00 & 81,00 & 50,00 \\
\hline Túnez (65\%) & 9,00 & 11,30 & 35,00 & 50,00 & 70,00 \\
\hline Togo (80\%) & 11,00 & 14,00 & 46,00 & 70,00 & 75,00 & 55,00 \\
\hline USA (75\%) & 10,18 & 13,10 & 27,50 & 42,00 & 55,00 \\
\hline Senegal (81\%) & 12,00 & 15,50 & 51,00 & 75,00 & 81,00 & 78,00 \\
\hline Jordania (70\%) & 10,00 & 12,00 & 36,00 & 45,00 & 59,00 & 50,00 \\
\hline Sahara (75\%) & nd & nd & 42,00 & 63,00 & 68,00 & 81,00 \\
\hline Sahara (80\%) & nd & nd & 50,00 & 75,00 & 81,00 \\
\hline
\end{tabular}

Tabla 3. Evolución de los precios del fosfato natural en varios países exportadores, 1970-1975 (en \% BPL y dólares por tonelada) (Anani Mensah, 2003; Zenaidi Karray, 1987; Ríos, 1989). Nota: 1 y 2: $1^{\text {er }}$ y $2^{\circ}$ semestre. ND: No hay datos.

Table 3. Evolution of natural phosphate prices in several exporting countries, 1970-1975 (\% BPL and dollars per ton) (Anani Mensah, 2003; Zenaidi Karray, 1987; Ríos, 1989). Note: 1 and 2: $1^{\text {st }}$ and $2^{\text {nd }}$ semester. ND: There are no data.

Desde el INI se opinaba que lo mejor para la empresa española era aliarse con OCP, por ser el mayor competidor en el mercado europeo y nacional, y llegar a acuerdos puntuales con IMC en aquellos mercados donde no se entrara en competencia directa con la empresa marroquí (América del Sur y Asia) (ASEPI, FOSBUCRAA, caja 44, doc. 3. Alfonso Álvarez Miranda a Presidente del INI. FosbucraaConversaciones con IMC. Madrid, 29 de abril de 1972). El problema con OCP era su pretensión de entrar en el accionariado de Fosbucraa y participar en todas las riquezas mineras del Sahara. Ante la negativa española, OCP cambió de estrategia y aceptó, en 1972, la creación de un cártel hispano-marroquí, fijando cuotas de producción y reparto de mercados. La estrategia marroquí era limitar el acceso del fosfato sahariano al mercado europeo e intentar restablecer la situación dominante de principios de los años sesenta. Ambas partes pusieron sobre la mesa sus respectivos programas de ventas para el quinquenio 1972-1976. Fosbucraa aspiraba a comenzar vendiendo un millón setecientas mil toneladas en 1973 para alcanzar los seis millones tres años más tarde. OCP, por su parte, planteaba unas previsiones iniciales de producción de catorce millones de toneladas hasta conseguir, en 1976, las dieciocho millones seiscientas mil toneladas. Para no incurrir en un exceso de oferta se acordó establecer una política común de precios y cuotas de mercado para Italia, Francia, Alemania y Rumanía, pero OCP se negó a que el fosfato sahariano se vendiera en Gran Bretaña, Benelux y Yugoeslavia. En cuanto al mercado español, ambas empresas fijaron toneladas y precios para 1973 y 1974. A Fosbucraa se le adjudicó una cuota de 500.000 toneladas para 1973 y de 750.000 toneladas para 1974. El resto, hasta completar una cifra cercana a los dos millones de toneladas, le correspondía a OCP (ASEPI, FOSBUCRAA, caja 44, doc. 4. Alfonso Álvarez Miranda a Claudio Boada (presidente del INI). Madrid, 29 de abril de 1972. Y, Nota Informativa 36/72. Informe visita a Rabat. Madrid, 26 de mayo de 1972).

La reacción de los clientes españoles de OCP (Unión Explosivos de Río Tinto, Industrias de Abonos de Navarra e Industrias Químicas Canarias, entre otros) (Enminsa, 1965; Arenas, 2014; Robles Teigeiro, 1992; Pérez Hernández, 2002) no se hizo esperar y amenazaron con incrementar las compras en Estados Unidos si subían los precios en relación a 1972. Las amenazas se cumplieron al año siguiente, cuando las empresas de fertilizantes comunicaron al gobierno su intención de repercutir en el precio final de sus productos los incrementos del coste del fosfato que se veían obligadas a comprar a OCP. En 1974, y en contra de los deseos del consejo de administración de Fosbucraa, el INI ordenó a la empresa española que diese prioridad a los suministros de mineral de fosfato al mercado nacional, limitando al mínimo las exportaciones al mercado exterior y dejando sin efecto aquellos compromisos en los que no hubiera penalizaciones, además de imponer un "precio político" por debajo del precio de mercado. Con esta decisión se rompía el acuerdo con OCP en el mercado nacional y se perdían mercados en el exterior. Si en 1974, el $72,5 \%$ del fosfato vendido por Fosbucraa se dirigió al mercado interior, en 1975 la cifra ascendió al 83.5\% (Tabla 4) (ASEPI, FOSBUCRAA, caja 575, exp. 1786. Nota informativa FBC-103. Situación y perspectiva del mercado de fosfato. Madrid, 20 de noviembre de 1973. Ibídem, caja 5583, doc. 16. Consejo de Administración. Acta $\mathrm{n}^{\circ} 159$. Madrid, 26 de marzo de 1974). 


\begin{tabular}{|c|c|c|c|c|}
\hline Año & $\begin{array}{c}\text { Mercado } \\
\text { Español }\end{array}$ & $\%$ & $\begin{array}{c}\text { Mercado } \\
\text { Mundial }\end{array}$ & $\%$ \\
\hline 1973 & 356 & 54.5 & 297 & 45.5 \\
\hline 1974 & 1.581 & 72.5 & 599 & 27.5 \\
\hline 1975 & 2.235 & 83.5 & 442 & 16.5 \\
\hline
\end{tabular}

Tabla 4. Exportaciones de fosfato de Fosbucraa, 1973-1975 (miles de toneladas y \%). (Elaboración propia a partir de Memorias y Balances de Fosbucraa).

Table 4. Fosbucraa's exports of phosphate, 1973-1975 (thousands of tons and \%) (Own elaboration from annual reports and balance sheets of Fosbucraa).

En 1975 el mercado reaccionó con una acusada caída de la demanda. Las repercusiones de la crisis mundial en la venta de fertilizantes en Europa provocaron una caída generalizada de los precios del fosfato en el primer semestre del año, que osciló entre el $15 \%$ y el $40 \%$ según los países. Los productores intentaron mantener los precios, pero la situación del mercado, en clara regresión, provocó la ruptura entre los principales productores (incluidos OCP y Fosbucraa), lo que se tradujo en una caída "desordenada de precios".

No hemos encontrado información suficiente que nos permita realizar un análisis pormenorizado de los costes de producción del mineral entre 1973 y 1975. Solo disponemos de algunos datos que nos ofrecen las memorias y balances de la empresa y de un informe remitido por Claudio Boada (presidente del INI) al ministro de Industria, José $\mathrm{M}^{\mathrm{a}}$ López de Letona, a finales de 1973. En base a estos datos, hemos estimado en 35 y en 47,5 dólares el coste FOB de una tonelada de fosfato para 1974 y 1975 respectivamente (Tabla 5).

\begin{tabular}{|l|c|c|}
\hline Concepto & 1974 & 1975 \\
\hline Personal & 7,8 & 10,6 \\
\hline Consumos & 5,7 & 7,7 \\
\hline Amortizaciones & 5,6 & 7,5 \\
\hline Cargas financieras & 13,3 & 18,0 \\
\hline Canon de superficie & 2,5 & 3,3 \\
\hline Impuesto Sociedades & 0,3 & 0,4 \\
\hline Total & 35,2 & 47,5 \\
\hline
\end{tabular}

Fuente: ASEPI, FOSBUCRAA, caja 575, exp. 1786. Claudio Boada a José Ma López de Letona. Madrid, 6 de diciembre de 1973.

Tabla 5. Coste FOB de la tonelada de fosfato de Bu-Craa, 1974-1975 (dólares por tonelada).

Table 5. FOB cost of the ton of phosphate from Bu-Craa, 1974-1975 (dollars per ton).
A lo largo de esos dos años la empresa exportó mayoritariamente fosfato de calidad $80 \%$ BPL. Si tenemos en cuenta que en el mercado exterior Fosbucraa aplicó los mismos precios que OCP y tomamos como referencia el coste de la tonelada de cada uno de esos dos años, tendremos que el precio medio de una tonelada en el mercado exterior osciló entre los 62,5 de 1974 y los 81 dólares de 1975, con unos márgenes bruto de beneficio por tonelada que rondaron los 27,5 en el primer año y los 33,5 dólares en el segundo. En el mercado nacional, por el contrario, la tonelada de fosfato se vendió a un precio inferior en 20 dólares al precio de mercado, con la que la empresa vio reducido su margen bruto de beneficio a 7,5 dólares la tonelada en 1974 y a 13,5 dólares en 1975. La gran beneficiada fue la industria de fertilizantes española que adquirió el fosfato a un precio medio que estuvo entre los 42,5 dólares la tonelada en 1974 y los 61 en 1975. De ahí las quejas de OCP que observaba como los embarques de fosfato marroquí en dirección al mercado español se redujeron en un $25 \%$.

Si exceptuamos el primer año de entrada en explotación de Fosbucraa, en el que el resultado del ejercicio fue negativo como consecuencia de la puesta a punto de las instalaciones y la escasa producción obtenida, la empresa fue rentable en los años 1974 y 1975 con unos beneficios brutos que alcanzaron los 877 millones de pesetas en 1974 y los 412 millones en 1975 (Tabla 6). La caída de los beneficios a la mitad en el último año se explica por la recesión del consumo de fosfato en el mercado mundial, por las dificultades económicas de los países consumidores y por la situación que se vivió en el Sahara Occidental (ASEPI, FOSBUCRAA, caja 575, exp. 1786. Claudio Boada a José $M^{a}$ López de Letona. Madrid, 6 de diciembre de 1973. Memoria y Balance del ejercicio 1975. Y CADN, Fond Rabat Ambassade 1956-1989. Pierre le Nail à M. le Ministre des Affaires Étrangères, $n^{\circ} 84$. Situation des phosphates au Maroc. Rabat, le 15 septembre 1976).

\begin{tabular}{|c|c|c|c|}
\hline Años & $\begin{array}{c}\text { Beneficios (millo- } \\
\text { nes pts) }\end{array}$ & $\begin{array}{c}\text { Rentabilidad eco- } \\
\text { nómica (\%) }\end{array}$ & $\begin{array}{c}\text { Rentabilidad } \\
\text { financiera (\%) }\end{array}$ \\
\hline 1973 & $-493,5$ & - & - \\
\hline 1974 & 876,9 & 3,1 & 9,7 \\
\hline 1975 & 412,2 & 1,4 & 4,3 \\
\hline
\end{tabular}

Tabla 6. Beneficios y rentabilidad de Fosbucraa, 1973-1975 (en millones de pts y \%) (Elaboración propia a partir de Memorias y Balances de Fosbucraa).

Table 6. Benefits and profitability of Fosbucraa, 1973-1975 (in millions pesetas and \%) (Own elaboration from annual reports and balance sheets of Fosbucraa). 


\section{Venta, cambio de nacionalidad y parálisis de la activi- dad minera, 1976-1983}

El 23 de enero de 1976, el presidente del INI, Juan Miguel Antoñanzas, y Mohamed Karim Lamrani, director de la Office Chérifien des Phosphates (OCP), de conformidad con lo recogido en la parte económica de los acuerdos de Madrid del 14 de noviembre de 1975 , formalizaron la venta del $65 \%$ de las acciones de Fosbucraa a la compañía estatal marroquí, por un valor de 5.850 millones de pesetas, a pagar en cuatro anualidades de $1.462,5$ millones de pesetas cada una. Durante las negociaciones que precedieron a la venta, Marruecos no aceptó comprar la totalidad de la sociedad, por lo que el INI, ante la obligación de seguir, optó por detentar el $35 \%$ de las acciones, ya que dicho porcentaje suponía el "tercio de bloqueo" (cualquier intento de ampliación de capital o disolución de la sociedad requería de su conformidad). En cuanto a los créditos que ya figuraban en el balance de la compañía, y que habían sido concertados por el Instituto con un consorcio de bancos extranjeros por valor de 213 millones de dólares (12.030 millones de pesetas) y de Fosbucraa con los bancos nacionales y proveedores, quedarían en las mismas condiciones en que fueron contratados y serían avalados de manera conjunta y solidaria por el INI (35\%), la OCP y el Banco Marroquí de Comercio Exterior (BMCE) (65\%). Lo mismo sucedía con los créditos concedidos por el Instituto a la empresa por un importe de 2.648 millones de pesetas, con la diferencia de que su amortización se efectuaría el 1 de julio de 1980, 1981 y 1982 (ASEPI, FOSBUCRAA, caja 5.736 , doc. 131. Contrato entre INI y OCP en relación con la venta de acciones de FOSBUCRAA. Madrid, 23 de enero de 1976).

El consejo de ministros celebrado el 6 de febrero ratificó el citado acuerdo y autorizó el cambio de nacionalidad y de domicilio social de la compañía. En la asamblea general extraordinaria celebrada en Rabat el 26 de mayo, el capital social de la empresa se mantuvo en los 5.000 millones de pesetas (328 millones de dírham) ya escriturados desde 1969, divididos en 500.000 acciones de 10.000 pesetas cada una, de las cuales 324.995 pertenecían a la OCP y el resto, 174.997, al INI (ASEPI, FOSBUCRAA, caja 5.736, doc. 26. El Ministro de Industria a Presidente del INI. Madrid, 25 de febrero de 1976). El contrato firmado entre ambas partes estipulaba una duración de 99 años, a contar desde la constitución de Enminsa en 1962, y el consejo de administración estaría presidido por Mohamed Karim Lamrani (presidente), Alfonso Álvarez Miranda (vicepresidente) y seis consejeros, dos españoles (Francisco Abellá Martín y José Manuel Fernández Felgueroso) y cuatro marroquíes
(ASEPI, FOSBUCRAA, caja 5736, doc. 99. Reunión de la Asamblea General Extraordinaria de PHOSBOUCRAA S.A., Rabat, 26 de mayo de 1976).

A finales de febrero, y coincidiendo con la salida definitiva de España del territorio, de los 2.574 trabajadores con los que contaba la empresa a 31 de diciembre de 1975 (1.624 españoles y 950 saharauis), sólo aceptaron quedarse 650 españoles y otros 650 autóctonos. Entre los españoles que no permanecieron, un $41 \%$ solicitó la indemnización y el otro $19 \%$ se acogió al cambio de empresa dentro del holding estatal. De los 300 saharauis que abandonaron la empresa, muchos se marcharon a luchar con la guerrilla del Frente Polisario, si bien no hay constancia de su número exacto (ASEPI, FOSBUCRAA, caja 5737, doc. 2. Consejo de Administración. Acta $n^{\circ} 186$. Madrid, 11 de marzo de 1976).

En los últimos años de la década de los setenta, la estrategia marroquí en relación con los fosfatos perseguía dos objetivos: por un lado, afianzar su participación en el mercado mundial de roca fosfática, incrementando las ventas en aquellos mercados donde más crecía la demanda y donde su presencia era insignificante (Europa Oriental) y, por el otro, la conversión de una tercera parte de su producción de fosfato natural en bienes intermedios, como el ácido fosfórico y el ácido sulfúrico, para su posterior exportación, en el marco de la industrialización dirigida en varios sectores estratégicos impulsada por el gobierno de Rabat.

En relación con el primer objetivo, y en un contexto mundial de caída de la demanda y de los precios, el reino alauita aprovechó la progresión de sus ventas en el mercado de Europa Oriental. Aunque la URSS era el segundo mayor productor mundial de roca fosfática, sus stocks empezaban a resentirse en la medida que las necesidades de fertilizante fosfatados crecían en los países del COMECON. Esta fue la razón que impulsó a Marruecos a firmar en 1978 un acuerdo económico con la URSS por un período de treinta años. Mediante este acuerdo, Moscú se comprometía a prestar asistencia técnica y financiera para poner en marcha la explotación del nuevo yacimiento de fosfato de Meskala, mientras que Rabat se mostraba dispuesto a suministrar mineral de fosfato a los países del Este, así como licencias de pesca para la flota soviética (ASEPI, FOSBUCRAA, caja 259, exp. A3-645, doc. 39. La estrategia marroquí en relación con los fosfatos. S.f., s.I.). El acuerdo de Meskala, bautizado por Hassan II como el "contrato del siglo", fue utilizado por éste como medio de presión ante la administración Carter para lograr un apoyo más firme por parte de Estados Unidos en la cuestión del Sahara occidental (Hernando de Larramendi, 1997). 
En lo que atañe a su segundo objetivo, el reino alauita se centró en relanzar el complejo químico de Safi, inaugurado a mediados de los años setenta, en el marco del plan de expansión de la OCP para el quinquenio 1973-1977. Ello implicaba ampliar su tamaño y su capacidad de producción con el fin de producir ácido sulfúrico, ácido fosfórico y fertilizantes complejos (NPK) (Oualalou, 1975; El Malki, 1989).

Tras un período de breve paralización de Fosbucraa en los meses finales de 1975, la explotación de la mina se interrumpió indefinidamente a partir del 6 de abril de 1976, como consecuencia del conflicto armado entre el ejército de Marruecos y el Frente Polisario que logró paralizar el centro minero con operaciones de sabotaje en la cinta transportadora y en la línea eléctrica Aaiún-Bucraa, además de la colocación de minas a lo largo del recorrido de la susodicha cinta y de los postes de suministro de energía. Entre 1976 y 1979 se hicieron Ilegar múltiples escritos y notas al Ministro de Industria y Energía, advirtiéndole de la difícil situación de la empresa y proponiendo la conveniencia de que el Instituto vendiera su participación en la misma.

En el verano de 1977, un informe intitulado: El futuro económico de Fosbucraa, ya describía a la empresa como una fuente de pérdidas importantes en los años venideros. Las razones esgrimidas eran tres. La primera se debía al aumento de los costes de producción, especialmente la mano de obra y los gastos relacionados con la conservación de los materiales. La segunda estaba relacionada con la caída del precio mundial del fosfato a partir de 1975. Entre ese año y 1977 (Figura 2), la cotización del fosfato mundial experimentó una caída del orden del $40 \%$. Aunque la tendencia cambió a finales de los setenta, los peores presagios se apoderaron del precio del fosfato que

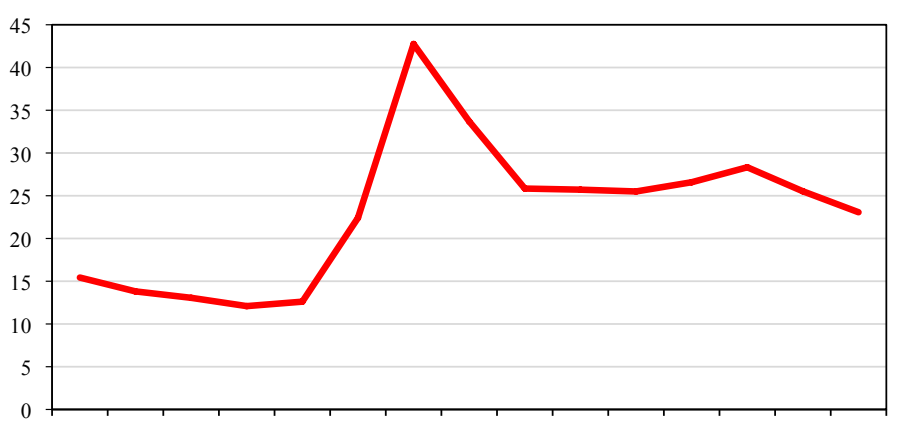

$\begin{array}{lllllllllllllll}1969 & 1970 & 1971 & 1972 & 1973 & 1974 & 1975 & 1976 & 1977 & 1978 & 1979 & 1980 & 1981 & 1982 & 1983\end{array}$

Figura 2. Precio del fosfato en el mercado internacional, 1969-1983 (dólares constantes de 1982) (Stowasser, 1989).

Figure 2. Price of phosphate in the international market, 1969-1983 (constant dollars in 1982) (Stowasser, 1989). volvió a descender otros 5,2 dólares entre 1981 y 1983, situándose en un nivel muy próximo al que tenía en 1974 (22.5 dólares). Con motivo de la caída del precio del fosfato, el presidente de Senegal, Leopold Sédar Senghor, convocó en Dakar, entre el 9 y el 11 de noviembre de 1976, una reunión de los países árabes y africanos productores de fosfato, a la que asistieron: Marruecos, Argelia, Túnez, Jordania, Togo y Senegal. En opinión del agregado comercial de la embajada francesa en Rabat, la conferencia tenía por objeto plantear una estrategia común para hacer frente a la política agresiva de precios practicada por las empresas de Estados Unidos en el mercado de Europa Occidental. Sin embargo, las diferencias existentes entre los asistentes en cuanto a la forma y naturaleza de la organización a crear, así como la hostilidad entre algunos de ellos (Marruecos y Argelia), dio al traste con la iniciativa senegalesa (CADN, Fond Rabat Ambassade 1956-1989. Pierre le Nail à M. le Ministre des Affaires Étrangères. Réunion à Dakar des représentants des pays africaines et arabes producteurs de phosphate. Dakar, le 15 novembre 1976).

El segundo shock petrolero de 1979 y el crecimiento de los desequilibrios macroeconómicos en los principales países consumidores, agravado con la revalorización del dólar en los primeros años del decenio de los ochenta, dio como resultado un incremento en los costes de producción del fosfato y la disminución de las importaciones de roca fosfática a causa del desequilibrio de la balanza comercial de los países importadores (Anani Mensah, 2003).

Si bien las dos razones anteriores eran importantes, la paralización de la actividad económica de la sociedad en un plazo que ya superaba los dos años fue determinante. La falta de seguridad debido al fracaso del ejército marroquí condicionaba todo. En 1978 el deterioro de las instalaciones ubicadas en la playa del Aaiún era alarmante. La central eléctrica y la potabilizadora tenían dos grupos diésel parados y uno en reparación. La línea eléctrica que conectaba con el Aaiún y con Bucraa tenía 17 postes averiados. De la cinta transportadora sólo funcionaban 5 de los 11 tramos y la planta de tratamiento de mineral estaba en revisión. Por si fuera poco, la situación del personal español comenzó a ser preocupante, ya que la mayoría de los operarios cualificados comenzaron a abandonar la empresa después de tres años de ver incumplidas "Ias promesas de una próxima reanudación de actividades". El factor psicológico jugó un papel importante. Entre enero de 1976 y mayo de 1979 , los explosivos colocados por el Polisario provocaron dos muertos y dos heridos de gravedad entre los trabajadores españoles. El deterioro de las insta- 
laciones por el ambiente corrosivo, insuficiente mantenimiento y falta de personal español cualificado no se vio frenado con la llegada de personal marroquí. A mediados de 1979, la empresa contaba con 1.405 empleados, de los que un $14,9 \%$ eran españoles, un $16.2 \%$ eran especialistas traídos de Marruecos y el $68.9 \%$ restante era "personal nativo". Ante la gravedad de la situación, los informes recomendaban al presidente del Instituto, una vez más, deshacerse cuanto antes de la participación en Fosbucraa (ASEPI, FOSBUCRAA, caja 259, exp. A3-645, doc. 41. Nota informativa C.S. 17/78. Situación Bucraa. Madrid, marzo de 1978).

La situación financiera de la empresa a finales de 1978 era insostenible. Las pérdidas de ese año ascendieron a 7.141 millones de pesetas (Figura 3), o sea, un $75 \%$ más que en el año anterior. A esa cifra habría que sumarle la cantidad de 6.181 millones de pesetas en concepto de amortizaciones no realizadas en los ejercicios de 1976 a 1978, lo que suponía un quebranto económico real de 13.322 millones de pesetas. Estas pérdidas se estaban cubriendo con créditos de tesorería facilitados por el accionista marroquí. A 31 de diciembre de 1978, el importe del crédito concedido por OCP a Fosbucraa se acercaba a los 11.000 millones de pesetas. El volumen de las citadas pérdidas estaba afectando de tal manera a la estructura financiera de la sociedad y pesaba tan fuertemente sobre las finanzas de OCP, que su presidente, Mohamed Karim Lamrani, estaba cada vez más convencido de la necesidad de abordar una ampliación de capital que corrigiera, en parte, la desproporción entre fondos propios $(26,6 \%$ del exigible total) y pasi-

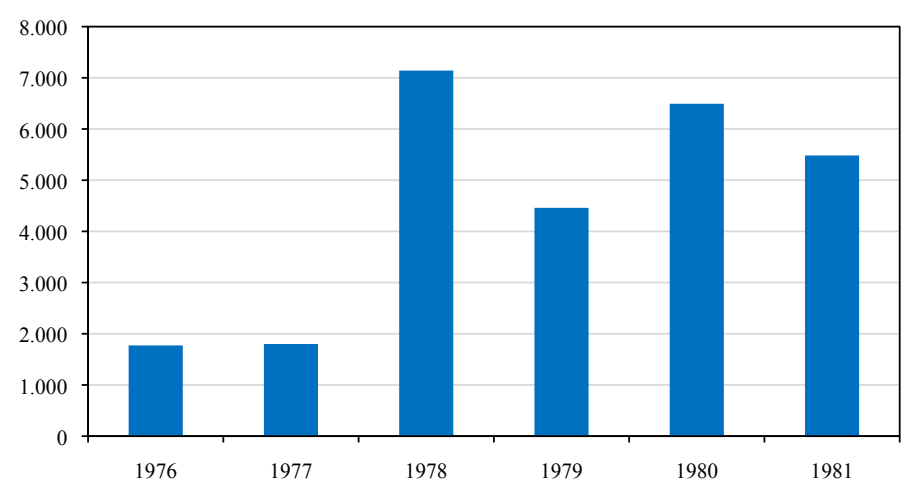

Fuente: Elaboración propia con datos de Martín Aceña y Comín (1991) y ASEPI, FOSBUCRAA, caja 259, exp. A3-645. El director de la División de Fertilizantes a Presidente del INI. Madrid, 15 de enero de 1982.

Figura 3. Pérdidas de Fosbucraa, 1976-1981 (millones de pts). Figure 3. Fosbucraa's losses, 1976-1981 (millions of pesetas). vo total. Es más, el pasivo a corto plazo que representaba el $51,2 \%$ del pasivo total en 1978 , aumentó al $64,8 \%$ a 31 de diciembre de 1981 .

Llovía sobre mojado. Ni la situación económica española ni la del Instituto eran las más adecuadas para soportar esas pérdidas. En los inicios de la transición, el INI había recuperado, por deseo de los gobernantes, su función de "hospital de empresas", absorbiendo a un gran número de compañías privadas al borde de la quiebra. A ello habría que añadir el que Fosbucraa se había convertido, en 1977, en la tercera sociedad del grupo con más pérdidas dentro del sector de la siderometalurgia y minería, superada sólo por Hunosa y Ensidesa (Martín Aceña and Comín, 1991).

En las notas e informes elevados a la dirección del Instituto en 1979 se contemplaban dos soluciones: o la venta del $35 \%$ de las acciones o la reducción progresiva de su participación accionarial en Fosbucraa. La primera era considerada como la más favorable para los intereses del INI. Con el fin de hacerla más creíble ante la parte marroquí, se abogaba por invertir en Marruecos el dinero que se obtuviera con la citada venta. La segunda consistía en transformar los créditos que el Instituto tenía avalados a Fosbucraa con vencimiento en los dos años siguientes (1980 y 1981), en un crédito a largo plazo a la OCP con garantía del tesoro marroquí y en condiciones de mercado. Para los responsables del INI esta solución era la menos deseada de las dos, ya que si el accionista mayoritario proponía llevar a cabo una ampliación de capital para consolidar la estructura financiera de la empresa y el Instituto no optaba por acudir, el INI iría perdiendo su participación en la misma proporción que aumentaría el capital por parte de OCP, lo que pondría en una situación muy comprometida al gobierno y al propio Instituto (ASEPI, FOSBUCRAA, caja 259 , exp. A3-645, doc. 47. Situación y perspectivas de Fosbucraa. Madrid, mayo de 1979).

Tanto el Ministerio de Asuntos Exteriores como el Ministerio de Industria y Energía eran partidarios de la segunda opción. Es más, desde el palacio de Santa Cruz (sede del MAE) se temía que una negativa marroquí a una propuesta de venta del paquete accionarial español, diera paso a una larga negociación en cuanto a la valoración de los activos de Fosbucraa, en la que el reino alauita se limitaría a ganar tiempo "sin ánimo real de permitir la salida española de la Empresa", hecho éste que ya estaba ocurriendo con las autoridades mauritanas en relación con los activos de Industries Mauritaniennes de Pêche (IMAPEC) (Martínez Milán, 2014). Todo lo que fuera eliminar obstáculos a la política de neutralidad en la cuestión saharaui y al mantenimiento de buenas relaciones 
con las partes en conflicto (Marruecos, por un lado, y Argelia con el Frente Polisario, por el otro), sería bien visto desde Asuntos Exteriores (ASEPI, FOSBUCRAA, caja 259, exp. A3-645, doc. 50. Ministro de Asuntos Exteriores a Ministro de Industria y Energía. Madrid, 27 de junio de 1979).

Ante la alarmante falta de actividad de la empresa, el INI suscribió un nuevo acuerdo el 11 de marzo de 1980 con Fosbucraa y OCP para resolver los problemas financieros de la sociedad. En dicho acuerdo se aceptaba un aplazamiento por 3 años del pago del principal pendiente e intereses del crédito de 213 millones de dólares, que en su parte proporcional del $65 \%$ tenía que hacer frente OCP, así como un aplazamiento también por 3 años del pago del principal más los intereses del crédito que el Instituto tenía concedido a Fosbucraa, y cuyo importe ascendía en esos momentos a 3.110 millones de pesetas. Por último, se acordó retrasar en 3 años el pago del principal e intereses de las entregas que por cuenta corriente venía realizando OCP desde 1976 para financiar la tesorería de Fosbucraa, con el compromiso de continuar haciéndolo hasta la normalización de la sociedad. Este acuerdo fue posible porque las partes entendían que en los tres años siguientes el centro minero alcanzaría un nivel de producción normal. Si esa situación no se daba se estableció la posibilidad de una reunión de los accionistas antes del 1 de enero de 1983 para encontrar una solución (ASEPI, FOSBUCRAA, caja 259, exp. A3-645, doc. 54. Acuerdo INIOCP. Madrid, 11 de marzo de 1980).

Con este acuerdo, Marruecos conseguía retener al gobierno español en el accionariado de la empresa y el Instituto conseguía paralizar cualquier intento de ampliación de capital, a la espera de que la situación en el territorio mejorara y la entrada en explotación de la mina le permitiera recuperar las cantidades adeudadas por Fosbucraa y OCP. Sin embargo, la situación, lejos de mejorar, empeoró. A finales de 1981, los fondos propios de la empresa representaban el $19,7 \%$ del pasivo total y las pérdidas acumuladas en los años 1980 y 1981, por valor de 11.976 millones, sobrepasaban en 413 millones de pesetas el capital social más las reservas (recursos propios) de la empresa. De ahí que en 1982 se volvió a repetir la situación creada a finales de la década de los setenta, en la que la sociedad necesitaba ampliar capital como única vía para sobrevivir (ASEPI, FOSBUCRAA, caja 259, exp. A3-645, doc. 66. Nota informativa C.S. 8/82. Fosbucraa en 1982. Madrid, 9 de enero de 1982).

La tendencia de las exportaciones del fosfato marroquí en los siete años que siguieron a 1975 tampoco ayudó mucho. Durante ese período, la evolución de las ventas atravesó por 3 fases diferentes: La pri- mera, entre 1976 y 1978, se caracterizó por un aumento del tonelaje exportado y una disminución de los ingresos. Esa tendencia cambió en 1979 con una ligera subida del precio del mineral de roca que perduró hasta 1981, y que coincidió con un descenso en las cantidades exportadas. En 1982 tanto el tonelaje como su valor cayeron. Durante ese año los fosfatos representaron el $27,3 \%$ del valor total de las exportaciones marroquíes frente al 55\% del año1975 (CADN, Fond Rabat Ambassade 1956-1989. Evolution des ventes de phosphates marocains au cours des dernières années. Janvier 1983).

En la reunión de la junta de accionistas de 1982, el representante de OCP manifestó que no podían seguir haciendo frente al mantenimiento de las necesidades de tesorería de Fosbucraa, rompiendo así el acuerdo alcanzado en marzo de 1980. Ante esta situación, el consejo de administración del INI acordó, previa autorización del Gobierno, solicitar a Fosbucraa el pago de la deuda en dólares, que en esos momentos ascendía a 123,7 millones (13.597 millones de pesetas), y en caso de que no fuera atendido por la empresa, proceder a la ejecución del crédito en base a las garantías dadas por OCP. Asimismo propuso renegociar la devolución del préstamo en pesetas que ascendía en aquél momento a 3.783 millones, concertando el reembolso del mismo a más largo plazo. Ante la falta de respuesta de Moncloa, el INI siguió insistiendo en pedir la ejecución de las garantías del crédito en dólares. La falta de colusión de intereses entre el Instituto y el Gobierno español era patente, ya que el INI se encontraba en una situación de bancarrota (Martín Aceña and Comín, 1991). Por si no fuera suficiente, las relaciones con los representantes de OCP se volvieron tensas. En un intento de desbloquear la situación, el consejo de administración del INI pidió al Gobierno español, el 13 de julio de 1983, la concesión de un crédito de Estado a Estado para que Fosbucraa pagara al Instituto la totalidad de la deuda (135 millones de dólares y 4.230 millones de pesetas, respectivamente), o bien los intereses de la deuda en dólares (53,7 millones) más la totalidad de la deuda en pesetas. En caso contrario, el INI seguiría insistiendo en ejecutar la garantía. El Gobierno español dio la callada por respuesta, tal vez con la esperanza de que la situación se normalizara (ASEPI, FOSBUCRAA, caja 572, exp. 1.771. El presidente del INI, Enrique Moya Francés, al Ministro de Industria y Energía. Situación financiera con relación al INI de Phosphates de BouCraa S.A. Madrid, 13 de abril de 1983).

Lo único que había mejorado era la situación de puesta a punto del centro minero. En Bu Craa se estaba trabajando con normalidad después de haber realizado trabajos de revisión a las dragalinas, en la plan- 
ta de tratamiento de mineral, así como en la cinta y en la zona de almacenamiento del fosfato. El consejo de administración de Fosbucraa concluyó que la empresa estaría preparada para entrar en funcionamiento a lo largo de 1982, estimando que el punto de equilibrio a partir del cual comenzaría a ser rentable su explotación sólo se alcanzaría con una producción de 4 o 5 millones de toneladas por año (ASEPI, FOSBUCRAA, caja 259, exp. A3-645, doc. 66. Reunión OCP-INI en Casablanca. Madrid, 26 de abril de 1982). El complejo minero se puso de nuevo en funcionamiento en el verano de ese año. Según el Servicio Geológico de Estados Unidos (Connor, 1984), la producción del yacimiento de Bu Craa en 1983 fue de 700.000 toneladas, muy lejos todavía del nivel de producción alcanzado en 1975. En opinión de Jeffrey Smith (2015), hasta 1998 la producción anual no superó el millón de toneladas, alcanzando en el 2006 el nivel que tenía en 1975.

Las dificultades económicas de Fosbucraa continuaron. En la asamblea general ordinaria celebrada el 30 de mayo de 1984 en Casablanca, se vivieron momentos de tirantes y situaciones dialécticas duras, haciendo caso omiso, la parte marroquí, de las alternativas ofrecidas por los representantes del Instituto para regularizar el pago de la deuda (ASEPI, FOSBUCRAA, caja 572, exp. 1771. El presidente del INI al Ministro de Industria y Energía. Madrid, 11 de junio de 1984). La situación se enquistó y la única solución para cubrir las pérdidas que había habido fue la ampliación de capital que se aprobó en 1996, a la que el INI no acudió, quedando reducida su participación accionarial al 12\%. En 2002, la Sociedad Española de Participaciones Industriales (SEPI), que había sucedido al Instituto, vendió las participaciones y abandonó el accionariado de la empresa (Gil Pecharromán, 2008).

\section{Conclusiones}

Fosbucraa fue el resultado de una apuesta clara y decidida del estado franquista para poner en marcha la explotación de un yacimiento de muy buena calidad, que volvió a convertir a España en un país productor y exportador de mineral de roca fosfática.

Aunque en algunos momentos no hubo colusión de intereses entre el consejo de administración de la empresa y el gobierno, los objetivos de producción y comercialización de Fosbucraa se habían conseguido o estaban próximos a hacerlo y la viabilidad de la empresa era una realidad cuando sobrevino la decisión política de abandonar el Sahara occidental.

La situación de guerra que se vivió en el territorio después de la salida española unido a la caída de la cotización internacional del fosfato, que se extendió hasta finales del siglo $\mathrm{XX}$, lastró el desarrollo de Fosbucraa. Aunque la adquisición de la mayor parte de las acciones de la sociedad le dio al reino alauita el control de la empresa y le permitió afianzar aún más su posición de primer exportador y tercer productor mundial de fosfato, la operación tuvo, a largo plazo, un elevado coste para OCP como consecuencia de las fuertes pérdidas habidas entre 1976 y 1981 que afectaron a la propia estructura financiera de la empresa. El Instituto, por su parte, consciente de que lo mejor para sus intereses era desprenderse de la sociedad, buscó diferentes opciones para que el socio mayoritario hiciera frente a su responsabilidad en una coyuntura de pérdidas millonarias para él y de discrepancias con el primer gobierno socialista a la hora de ejecutar la garantía de la deuda. Los intereses políticos y económicos españoles en Marruecos predominaron sobre lo demás y hubo que esperar a los años finales del siglo pasado (1996) para realizar una reestructuración de capital en la que OCP se hizo con el $88 \%$ del paquete accionarial, tomando el control definitivo seis años más tarde.

\section{Agradecimientos}

Este trabajo se ha beneficiado de los proyectos de investigación HAR2012-36414 y HAR2014-56428-C3-3$P$ (subproyecto Perceval), financiados por el Ministerio de Economía y Competitividad. Agradezco asimismo los comentarios y sugerencias hechos por Antonio Escudero a una versión preliminar de este artículo, así como los realizados por los evaluadores anónimos del Boletín Geológico y Minero. No obstante, cualquier error que contenga el mismo es responsabilidad única de su autor.

\section{Referencias}

Anani Mensah, A., 2003. Dynamique et comportements stratégiques sur le marché international du phosphate. Mondes en Développement, vol. 31 (122), 37-56. DOI 10.391/med.122.0037

Arenas Posadas, C., 2014. Río Tinto: un mito minero en declive (1954-2003). Comunicación presentada al XI Congreso Internacional de la Asociación Española de Historia Económica (AEHE), CUNEF, Madrid.

Bárbulo, T., 2002. La historia prohibida del Sahara español. Ediciones Destino, Barcelona, 347 pp.

Camprubí, L., 2015. Resource Geopolitics: Cold War Technologies, Global Fertilizers, and the Fate of Western Sahara. Technology and Culture, vol. 56 (3), 676-703.

Connor, K. 1984. The Mineral Industry of Morocco. Minerals 
Yearbook, pp. 575-584. http://minerals.usgs.gov/minerals/pubs/usbmmyb.html

Cordero Olivero, I., and Lemus López, E. 2015. La cuestión del Sahara: una vision desde el "Quai d'Orsay". Ayer 99 (3), 123-148.

Dalle, I. 2004. Les Trois Rois. La monarchie marocaine de I'indépendance à nos jours. Paris, Fayard, 840 pp.

Díaz Morlán, P. 2015. Empresarios, militares y políticos. La Compañía Española de Minas del Rif (1907-1967). Marcial Pons Historia-Universitat d'Alacant, 232 pp.

El Malki, H. 1989. Trente ans d'économie marocaine, 19601900. CNRS, Paris, 248 pp.

Enminsa. 1965. Industria de fertilizantes fosfatados en España. Kabana, Madrid, 352 pp.

Gil Pecharromán, J. 2008. Entre Hendaya y El Aiún. La política exterior del Franquismo (1939-1975). Flor del Viento Ediciones, Barcelona, 413 pp.

Hatton, G. 2009. Les enjeux financiers et économiques du protectorat marocain (1936-1956) : politique publique et investisseurs privés. Société Française d'Histoire d'Outre-Mer, Paris, 445 pp.

Hernando de Larramendi, M. 1997. La política exterior de Marruecos. Editorial Mapfre, Madrid, 469 pp.

Martín Aceña, P. and Comín, F. 1991. INI, 50 años de industrialización en España. Espasa Calpe, Madrid, 542 pp.

Martínez Milán, J. M. 2014. Las inversiones del Estado español en la industria derivada del pescado en el área sahariano-mauritana, 1946-1980. Revista de Historia Industrial, 54 (1), 117-137.

Martínez Milán, J. M. 2017. La larga puesta en escena de los fosfatos del Sahara Occidental, 1947-1969. Revista de Historia Industrial, 69 (monográfico 4), 177-205. Oualalou, F. 1975. La politique phosphatière marocaine et les tentatives d'adaptation aux variations de la demande internationale. Annuaire de l'Afrique du Nord, CNRS-CRESM, Paris, vol. 13, 41-70.

Pardo Sanz, R. 2007. El proceso de descolonización. In: Oreja, M. and Sánchez, R. (ed.), Entre la historia y la memoria. Fernando María Castiella y la política exterior de España, 1957-1969. Real Academia de Ciencias Morales y Políticas, Madrid, 83-134.

Pérez Hernández, C. 2002. Industrias Químicas Canarias, S.A. (1940-1983). Un ejemplo de la tentativa industrializadora de las islas. In: Carnero Lorenzo, F., Nuez Yánez (Coord.). Empresa e Historia en Canarias. Fundación Fydes - Caja Canarias, Tenerife, 153-175.

Piniés, J. 1990. La descolonización del Sáhara: un tema sin concluir. Espasa Calpe, Madrid, 236 pp.

Ríos, J.M. 1989. ¡iSahara!! ¡¡Sahara!!. La aventura de los fosfatos, un episodio inédito. Fundación Gómez Pardo, Madrid, $311 \mathrm{pp}$.

Rodríguez Jiménez, J. L. 2015. Agonía, traición, huida. El final del Sahara Español. Crítica, Barcelona, 676 pp.

Robles Teigeiro, L. 1992. La industria de fertilizantes nitrogenados y fosfatados: una perspectiva histórica (19391989). Revista de Estudios Agro-Sociales, 161, 189-211.

Smith, J. 2015. The Taking of the Sahara: the role of natural resources in the continuing occupation of Western Sahara. Global Change, Peace \& Security, 27 (3), 263284.

Stowasser, W. F. 1989. Phosphate Rock Minerals Yearbook. Minerals Yearbook, pp. 769-787. http://minerals.usgs.gov/ minerals/pubs/usbmmyb.html.

Zenaidi Karray, B. 1987. Les marchés mondiaux des phosphates et des engrais phosphates. Economica, Paris, 288 pp.

\section{FUENTES DOCUMENTALES}

Centro de Documentación y Archivo Histórico de la Sociedad Estatal de Participaciones Industriales en Madrid. FOSBUCRAA, cajas: 4515, 45, 4728, 4904, 5557, 2383, 5383, 44, 575, 259, 5736, 5737 y 572.

Centre des Archives Diplomatiques de Nantes. Fond Rabat Ambassade (558PO/1/156), 1956-1989.

Recibido: octubre 2016

Revisado: marzo 2017

Aceptado: mayo 2017

Publicado: diciembre 2017 